\title{
Analysis of Influence of Key Parameter $\mu$ on the Performance of Levenberg - Marquardt Based Iteration Square Root Cubature Kalman Filter
}

\author{
Jing $\mathrm{Mu}^{1, \mathrm{a}}$ and Changyuan Wang ${ }^{1, \mathrm{~b}}$ \\ ${ }^{1}$ School of Computer Science and Engineering, Xi'an Technological University, Xi'an, 710032, China \\ amujing1977@163.com, byw901@163.com
}

Keywords: Nonlinear filtering; Levenberg - Marquardt method; Re-entry ballistic targets

\begin{abstract}
The key parameter $\mu$ has large influence on the performance of L-M method based Iteration square root cubature Kalman filter (ISRCKFLM) proposed to improve the low state estimation accuracy of nonlinear state estimation due to large initial estimation error and nonlinearity of measurement equation. We analyze the impact of the key parameter on performance of the ISRCKFLM algorithm for re-entry ballistic target tracking; and find the interval of the key parameter suitable for estimation accuracy and faster convergence speed.
\end{abstract}

\section{Introduction}

Recently, one type of suboptimal nonlinear filters based on numerical multi-dimensional integral were introduced in [1], such as cubature Kalman filter (CKF), which used cubature rule to approximate the recursive Bayesian estimation integrals under the Gaussian assumption. The CKF can solve high-dimensional nonlinear filtering problems with minimal computational effort. Furthermore, the stability of CKF for non-linear systems with linear measurement is analyzed and the certain conditions to ensure that the estimation error of the CKF remains bounded are proved in [2]. Moreover, some usage of CKF are reported; for example, exploiting CKF in process monitoring in [3], the cubature rule in the structure of unknown input observer to achieve robust sensor fault detection [4], and using the square-root cubature information filter (SCIF) to deal with the problem of multi-target tracking in a distributed camera network in [5].

However, the implementation of CKF need compute the Cholesky factors of the error covariance evaluated and generate ill effects that may eventually lead to an unstable or even divergent behavior. In order to mitigate ill effects and improve the numerical stability, the cubature rule is exploited as square root cubature information filter [6] and the square root cubature Kalman filter (SRCKF) was developed in [1]. On the other hand, because of the large initial error and measurement error in the applications of state estimation for re-entry ballistic target with unknown ballistic coefficient, the SRCKF also shows its weakness in the robustness and estimation accuracy. L-M method based iteration cubature Kalman filter (ICKFLM) is developed in [7]. As we know, Levenberg-Marquardt (abbr. L-M) method has the same global convergence as Gauss-Newton method, and adjusting key parameter $\mu$ in the L-M method accelerates the convergence speed. In order to improve the numerical stability, we develop the L-M method based Iteration square root cubature Kalman filter (ISRCKFLM).

The rest of the paper is organized as follows. We begin in Section 2 with a description of L-M method based Iteration square root cubature Kalman filter (ISRCKFLM). Then we analyze and discuss the influence of key parameter $\mu$ on the performance of ISRCKFLM algorithm to track re-entry ballistic target (RBT) with unknown ballistic coefficient in Section 3 . Finally, Section 4 concludes the paper.

\section{Levenberg - Marquardt Method Based Iteration Square Root Cubature Kalman Filter}

Consider the following nonlinear dynamics system: 


$$
\begin{aligned}
& \boldsymbol{x}_{k}=\boldsymbol{f}\left(\boldsymbol{x}_{k-1}\right)+\boldsymbol{w}_{k-1} \\
& z_{k}=\boldsymbol{h}\left(\boldsymbol{x}_{k}\right)+\boldsymbol{v}_{k}
\end{aligned}
$$

where $\boldsymbol{f}$ and $\boldsymbol{h}$ are some known nonlinear functions; $\boldsymbol{x}_{k} \in \square^{n_{x}}$ and $\boldsymbol{z}_{k} \in \square^{n_{z}}$ is state and the measurement vector, respectively; $\boldsymbol{w}_{k-1}$ and $\boldsymbol{v}_{k}$ are process and measurement Gaussian noise sequences with zero means and covariance $\boldsymbol{Q}_{k-1}$ and $\boldsymbol{R}_{k}$, respectively, and $\left\{\boldsymbol{w}_{k-1}\right\}$ and $\left\{\boldsymbol{v}_{k}\right\}$ are mutually uncorrelated.

Suppose that the state distribution at $k-1$ time is $\mathbf{x}_{k-1}: \mathrm{N}\left(\hat{\mathbf{x}}_{k-1}, \mathbf{S}_{k-1} \mathbf{S}_{k-1}^{T}\right)$, Levenberg - Marquardt based Iteration square root cubature Kalman filter (ISRCKFLM) is developed based on Reference [7] as follows.

(1) Time Update

1) Calculate the cubature points and propagate the cubature points through the state equation

$$
\begin{aligned}
& \boldsymbol{X}_{i, k-1}=\boldsymbol{S}_{k-1} \boldsymbol{\xi}_{i}+\hat{\boldsymbol{x}}_{k-1} \\
& \boldsymbol{X}_{i, k}^{*}=\boldsymbol{f}\left(\boldsymbol{X}_{i, k-1}\right)
\end{aligned}
$$

where $\xi_{i}=\sqrt{m / 2}[1]_{i}, \omega_{i}=1 / m, i=1, \cdots m=2 n_{x}$, the $[1]_{i}$ is a $n_{x}$ dimensional vector and is generated according to the way described in [2].

2) Evaluate the predicted state and square root of the predicted covariance

$$
\overline{\boldsymbol{x}}_{k}=\sum_{i=1}^{m} \omega_{i} \boldsymbol{X}_{i, k}^{*}
$$

$$
\bar{S}_{k}=\operatorname{Tria}\left(\left[\chi_{k}^{*} S_{Q, k-1}\right]\right)
$$

here, $\boldsymbol{S}_{Q, k-1}$ denotes a square-root factor of $\boldsymbol{Q}_{k-1}$ and Tria() is denoted as a general triagularization algorithm. The matrix $\chi_{k}^{*}$ is defined as:

$$
\chi_{k}^{*}=1 / \sqrt{m}\left[\boldsymbol{X}_{1, k}^{*}-\overline{\boldsymbol{x}}_{k} \boldsymbol{X}_{2, k}^{*}-\overline{\boldsymbol{x}}_{k}, \cdots, \boldsymbol{X}_{m, k}^{*}-\overline{\boldsymbol{x}}_{k}\right]
$$

3) Evaluate the modified covariance:

$$
\tilde{\boldsymbol{P}}_{k}=\left[\boldsymbol{I}-\overline{\boldsymbol{S}}_{k} \overline{\boldsymbol{S}}_{k}^{T}\left(\overline{\boldsymbol{S}}_{k} \overline{\boldsymbol{S}}_{k}^{T}+\frac{1}{\mu_{i}} \boldsymbol{I}\right)^{-1}\right] \overline{\boldsymbol{S}}_{k} \overline{\boldsymbol{S}}_{k}^{T}
$$

where $\mu_{i}$ is adjusting parameter.

(2) Measurement update

1) Set the initial value as: $\hat{\boldsymbol{x}}_{k}^{(0)}=\overline{\boldsymbol{x}}_{k}$.

2) Assuming the $I$ - th iterate $\hat{\boldsymbol{x}}_{k}^{(i)}$, calculate the matrix

$$
\boldsymbol{L}_{k}^{(i)}=\tilde{\boldsymbol{P}}_{k} \boldsymbol{J}_{h}^{T}\left(\hat{\boldsymbol{x}}_{k}^{(i)}\right)\left[\boldsymbol{J}_{h}\left(\hat{\boldsymbol{x}}_{k}^{(i)}\right) \tilde{\boldsymbol{P}}_{k} \boldsymbol{J}_{h}^{T}\left(\hat{\boldsymbol{x}}_{k}^{(i)}\right)+\boldsymbol{R}_{k}\right]^{-1}
$$

3) Calculate the $i$-th iterate

$$
\hat{\boldsymbol{x}}_{k}^{(i+1)}=\overline{\boldsymbol{x}}_{k}+\boldsymbol{L}_{k}^{(i)}\left\{z_{k}-\boldsymbol{h}\left(\boldsymbol{x}_{k}^{(i)}\right)-\boldsymbol{J}_{h}\left(\hat{\boldsymbol{x}}_{k}^{(i)}\right)\left(\overline{\boldsymbol{x}}_{k}-\hat{\boldsymbol{x}}_{k}^{(i)}\right)\right\}-\mu_{i}\left\{\boldsymbol{I}-\boldsymbol{L}_{k}^{(i)} \boldsymbol{J}_{h}\left(\hat{\boldsymbol{x}}_{k}^{(i)}\right)\right\} \tilde{\boldsymbol{P}}_{k}\left(\overline{\boldsymbol{x}}_{k}-\hat{\boldsymbol{x}}_{k}^{(i)}\right)
$$

4) Calculate the iteration termination condition

$$
\left\|\boldsymbol{x}_{k}^{(i+1)}-\boldsymbol{x}_{k}^{(i)}\right\| \leq \varepsilon \text { or } i=N_{\max }
$$

$\varepsilon$ and $N_{\max }$ are predetermined threshold and maximum iterate number, respectively. If the termination condition meets, the iterate return to 5); otherwise continue to 2).

5) Calculate the state estimation at $k$ time instant 
$\hat{\boldsymbol{x}}_{k}=\hat{\boldsymbol{x}}_{k}^{(N)}$

6) Evaluate the cross-covariance and square root of innovation covariance at $k$ time

$$
\begin{aligned}
& \boldsymbol{P}_{x z}=\overline{\boldsymbol{S}}_{k} \overline{\boldsymbol{S}}_{k}^{T} \boldsymbol{J}_{h}^{T}\left(\hat{\boldsymbol{x}}_{k}^{(N)}\right) \\
& \boldsymbol{S}_{z z}=\operatorname{Chol}\left(\left[\boldsymbol{J}_{h}\left(\hat{\boldsymbol{x}}_{k}^{(N)}\right) \overline{\boldsymbol{S}}_{k} \quad \boldsymbol{S}_{R, k}\right]\right)
\end{aligned}
$$

7) Calculate the square root of covariance at $k$ time

$$
\begin{aligned}
& \boldsymbol{K}_{k}=\boldsymbol{P}_{x z} / \boldsymbol{S}_{z z}^{T} / \boldsymbol{S}_{z z} \\
& \boldsymbol{S}_{k}=\operatorname{Chol}\left(\left[\overline{\boldsymbol{S}}_{k}-\boldsymbol{K}_{k} \boldsymbol{J}_{h}\left(\hat{\boldsymbol{x}}_{k}^{(N)}\right) \overline{\boldsymbol{S}}_{k} \quad \boldsymbol{K}_{k} \boldsymbol{S}_{R, k}\right]\right)
\end{aligned}
$$

where symbol "/ " represents the matrix right divide operator.

The sequences obtained using Eq.10 have the global convergence. And tuning the key parameter $\mu$ accelerates its convergence rate.

\section{Influence of $\mu$ on the Performance of ISRCKFLM Algorithm with Applications to Re-Entry Ballistic Target Tracking}

Now we analyze the impact of the key parameter $\mu$ on the performance of ISRCKFLM algorithm with applications to the re-entry ballistic target tracking. In the simulation, model of target motion and of radar measurements of re-entry ballistic target with unknown ballistic coefficient are established in [8]. And the parameters and the initial state estimate are the same as in [8]. To demonstrate the performance of the ISRCKFLMs with various key parameters $\mu$, we still use the root-mean square error (RMSE) and average accumulated mean-square root error (AMSRE) in the position, velocity and ballistic coefficient introduced in [8]. All the simulations were done in MATLAB on a ThinkPad PC with an Intel (R) CORE i5 M480 processor with the $2.67 \mathrm{GHz}$ clock speed and 3GB physical memory. All performance curves and figures in this subsection were obtained by averaging over 100 independent Monte Carlo runs. All the filters are initialized with the same condition in each run.

In order to analyze the influence of the key parameter $\mu$ on the performance of the ISRCKFLM algorithm, we apply the ISRCKFLM with the various values of $\mu$ to state estimation for re-entry ballistic target. The mark of ISRCKFLM with various values of $\mu$ is listed in Table. 1. Fig. 1-Fig. 3 shows the RMSEs of SRCKF and ISRCKFLMs with various values of $\mu$ in position, velocity and ballistic coefficient. And Table. 2 shows the AMSREs in position, velocity and ballistic coefficient for SRCKF and ISRCKFLMs with various values of $\mu$.

Table 1 LISRCKF's mark with various values about $\mu$

\begin{tabular}{|c|c|c|c|}
\hline Various values of $\boldsymbol{\mu}$ & ISRCKFLM's mark & Various values of $\boldsymbol{\mu}$ & ISRCKFLM's mark \\
\hline$\mu_{1}=1.2 \times 10^{-4}$ & ISRCKFLM $\mu_{1}$ & $\mu_{4}=10^{-6}$ & ISRCKFLM $\mu^{4}$ \\
\hline$\mu_{2}=10^{-4}$ & ISRCKFLM $\mu_{2}$ & $\mu_{5}=10^{-10}$ & ISRCKFLM $\mu 5$ \\
\hline$\mu_{3}=10^{-5}$ & ISRCKFLM $\mu_{3}$ & & \\
\hline
\end{tabular}

From Fig. 1 - Fig. 3 and Table. 2, the RMSE and ARSME in position and velocity for the ISRCKFLM with $\mu_{1}=1.2 \times 10^{-4}$ begin to marginally decrease compared to those of SRCKF. The RMSEs and AMSREs of ISRCKFLM with $\mu_{2}=10^{-4}$ in position, velocity and ballistic coefficient significantly reduce, but the ISRCKFLM algorithm has very slow convergence rate, and the RMSEs and AMSREs of ISRCKFLM with $\mu_{3}=10^{-5}$ largely decrease, moreover, has faster convergence rate compared to those of ISRCKFLM with $\mu_{2}=10^{-4}$. Meanwhile, we can see that the RMSEs and AMRSEs of ISRCKFLM in position, velocity and ballistic coefficient are almost at the same level when $\mu$ is selected as $\mu=10^{-5}, 10^{-6}, 10^{-10}$. 
Through a large number of simulation experiments and analysis, the ISRCKFLM significantly decrease the estimation error and has faster convergence speed when the key parameter $\mu$ is selected in the interval of $0<\mu<10^{-5}$.

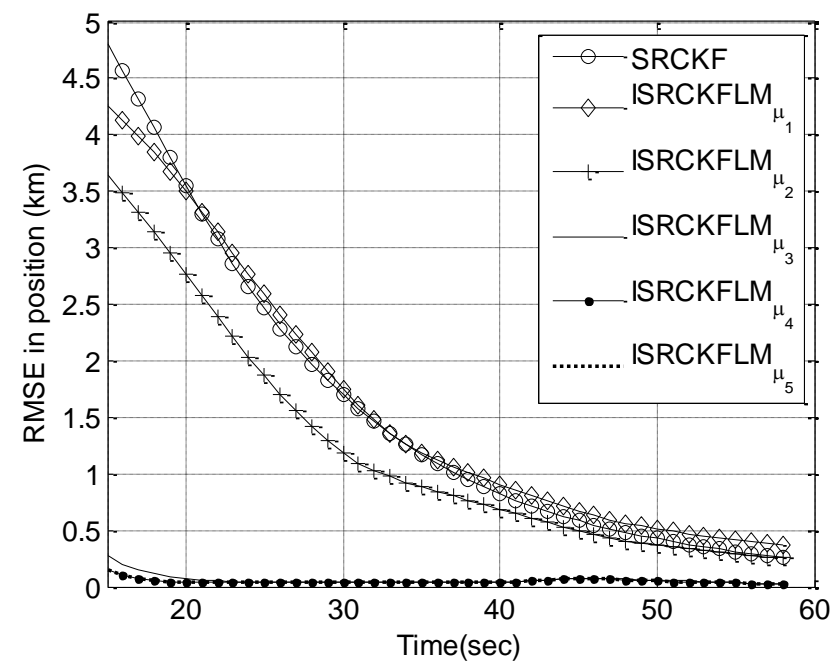

Figure 1. RMSEs of ISRCKFLM with various values of $\mu$ in position

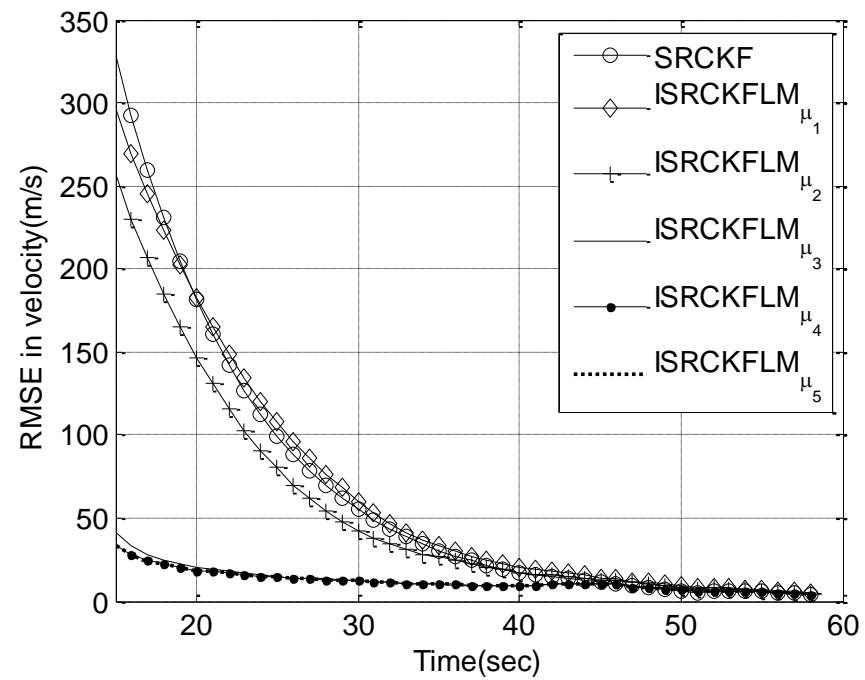

Figure 2. RMSEs of ISRCKFLM with various values of of $\mu$ in velocity

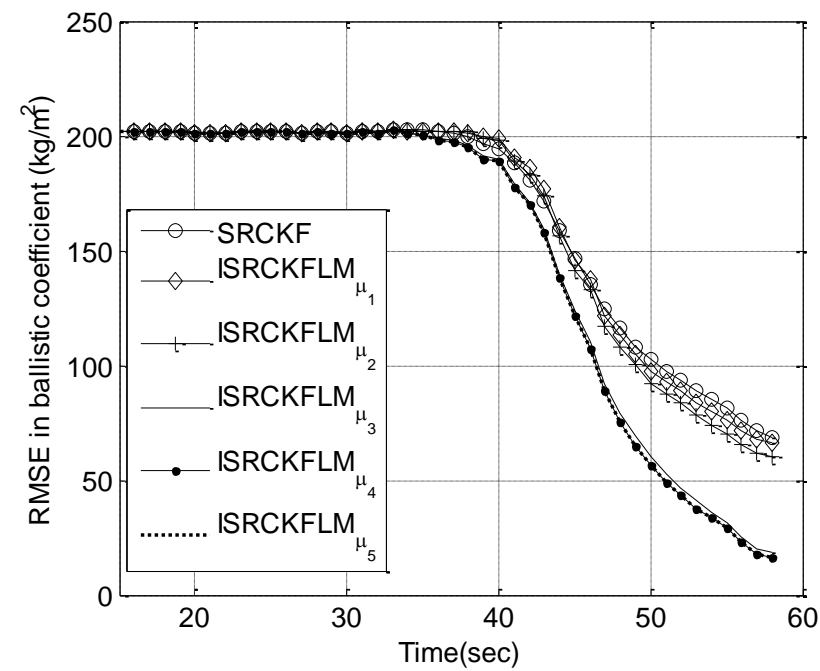

Figure 3. RMSEs of ISRCKFLM with various value of $\mu$ in ballistic coefficient 
Table 2 AMRSEs of ISRCKFLMs with various $\mu$ 's values in position, velocity and ballistic coefficient

\begin{tabular}{|c|c|c|c|}
\hline Various filters & $\operatorname{ARSME}_{p}(\mathbf{m})$ & ARSME $_{v}(\mathbf{m} / \mathbf{s})$ & ARSME $_{\beta}\left(\mathbf{k g} / \mathbf{m}^{2}\right)$ \\
\hline SRCKF & 2693.756 & 305.778 & 134.108 \\
\hline ISRCKFLM $\mu 1$ & 2477.752 & 287.356 & 135.174 \\
\hline ISRCKFLM $\mu p$ & 2129.194 & 275.345 & 134.106 \\
\hline ISRCKFLM $\mu \beta$ & 891.914 & 222.033 & 129.882 \\
\hline ISRCKFLM $\mu$ & 861.886 & 220.083 & 129.633 \\
\hline ISRCKFLM $\mu 5$ & 860.782 & 220.004 & 129.629 \\
\hline
\end{tabular}

\section{Conclusion}

In this study, the impact of key parameter $\mu$ on the performance of ISRCKFLM is analyzed. We make a lot of simulations and find that the key parameter $0<\mu<10^{-5}$ selected is the most suitable for the better performance of the ISRCKFLM. This demonstrates deeply that the ISRCKFLM algorithm is much more effective and improves the performance of state estimation to a marked degree and has the fast convergence rate.

\section{Acknowledgements}

The authors would like to thank the support of Special fund of Educational Department of Shaanxi Province under Grant 14JK1347 and the National Natural Science Foundation of China under Grant 61308120.

\section{References}

[1] 1Arasaratnam, I.; Haykin, S. Cubature Kalman filters. IEEE T. Automat. Contr. 2009, 54 (6), pp. 1254-1269.

[2] 6Jafar, Z.; Ehsan, S. Convergence analysis of non-linear filtering based on cubature Kalman filter. IET Sci. Meas. Technol. 2015, 9 (3), pp. 294-305.

[3] 7Zarei, J.; Shokri, E. Nonlinear and constrained state estimation based on the cubature Kalman filter, Ind. Eng. Chem. Res.2014, 53 (10), pp. 3938-3949.

[4] 8Zarei, J.; Shokri, E. Robust sensor fault detection based on nonlinear unknown input observer, Measurement. 2014, 48, pp.355-367.

[5] 10Chen, Y.M.; Zhao, Q.J. A Novel Square-Root Cubature Information Weighted Consensus Filter Algorithm for Multi-Target Tracking in Distributed Camera Networks. Sensors. 2015, 15, pp. 10526-10546.

[6] 12Chandra, K.P.B.; Da-Wei, G; Postlethwaite, I. Square root cubature information filter, IEEE Sens. J. 2013, 13 (2), pp. 750-758.

[7] 13Mu. J.; Cai. Y.; Wang. C.Y. L-M Method Based Iteration Cubature Kalman Filter and its Applications, Journal of Xi' an Technological University, 2013, 33 (1):1-6.

[8] 14Mu. J.; Cai. Y. Likelihood-based iteration square - root cubature Kalman filter with applicaitons to state estimation of re-entry ballistic target. T I. Meas. Control. 2013, 35(7), pp. 949-958. 